\title{
Pix2Trips - a system supporting small groups of urban tourists
}

\author{
Halina Kwasnicka \\ Wroclaw University of Science and Technology \\ wyb. Wyspianskiego 27, 50-370 Wroclaw, Poland \\ Email: halina.kwasnicka@pwr.edu.pl
}

\author{
Tsvetan Ovedenski \\ Chaos Group \\ Bulgaria
}

Email: tsvetan@tsovedenski.com,ccovedenski@gmail.com

\begin{abstract}
Group recommendation systems are the subject of many publications, but still is a gap between research results and group decision support systems' needs. Tourists often do not know which attractions they would like to visit. The Pix2Trips system asks the group's members to indicate images that they would like. On this basis, Pix2Trips models the group's preferences and adjusts them to the proposed places' models. Some tourist places in Wroclaw city, Poland, were used in experiments. The paper presents the system's components and discusses the results of the experiments. Conclusions indicate the good overall evaluation of the Pix2Trips system and further research.
\end{abstract}

\section{Problem StATEMEnT}

$\mathbf{L}$ ET us imagine that we want to visit monuments in a city in a group of several people. We need to make a list of places to visit so that all group members are reasonably satisfied. The decision about the places worth visiting requires a meeting and discussion within the group, but we can unknown all potentially interesting places in a new city. We aim to develop a computer system targeting groups of city tourists which should enable remote cooperation. In the proposed system, users define their preferences only by selecting multiple images. It means that the entire group's recommendation is defined based on the individual preferences indicated by chosen images. Our goal is to check if this method combined with an aggregation strategy will work for small groups. The research question is: how different aggregation strategies, including two proposed by authors, influence the quality of recommendations? Section 2 presents an overview of Group Recommender Systems. Section 3 describes the proposed Pix2Trips system. In the 4th section, we briefly discuss experiments with artificial and real groups. Conclusions end the paper.

\section{GRoup RECOMMENDER SySTEMS (GRS)}

Group Decision Support Systems (GDSS) extend Decision Support Systems (DSSs) with groupware functionality and various combination preferences strategies. The popular classification of groupware considers group proximity and time of communication [1]. The system can be targeted at groups located in:

- same place and same time (difficult in larger groups [2]),

- same place and different time,

- different place and the same time.

- different place and different time.

Expanding recommended systems to support multiple users requires that either profiles or recommendations be merged [3]. Eight different strategies are presented in [4]:
1) Average - averages individual values

) Average without misery - averages values below a threshold

3) Multiplicative - multiplies individual values,

4) Least misery - takes the minimum of individual values,

5) Most pleasure - takes the maximum of individual values,

6) Approval voting - counts values above a threshold,

7) Borda counts - assigns positions to values and sums them up,

8) Dictatorship - takes values of the most respected individual.

The effect of group dynamics is usually ignored in literature [5]. To solve the cold start problem, i.e., when we do not have information about a new user, two methods are used: one traditional using the questionnaire and the second - picturebased. We focus on the second one. Authors of [6] proposed picture-based elicitation where users select pictures as a base for profile determining. They use a Seven-Factor model based on the analysis Big Five personality factors [7], and 17 tourist roles [8] representing short-term preferences. The seven factors are the following: (1)sun-loving and connected, (2)educational, (3)independent, (4)culture-loving, (5)open-minded and sportive, (6)risk-seeking, (7)nature and recreation. The needed data is gathered in two steps: (1)each picture is assigned one factor as the most relevant, (2)the recommendation base is built manually, with experts' help. Authors of [9] tried to automate this tedious and subjective task via text-mining techniques. Paper [10] proposes another way of mapping images to tourist types: they defined 17 tourist roles and the tourism-related images. In [11] a user selects photos in several rounds: in subsequent rounds, presented pictures are refined based on the previous choices. The functionality of Groupware is connected with the size and proximity of target groups. INTRIGUE [13] targets large heterogeneous groups of unfamiliar people. In contrast, STSGroup [14] and Hootle+ [15] are more suitable for small groups of family or friends.

\section{Pix2Trips - THE PROPOSED SYSTEM}

The proposed system is dedicated to small groups of tourists to suggest interesting places in a new city. Users do not complete any questionnaire or provide personal data. The system asks the user to indicate which objects from pictures presented to him he would like to visit. On this basis, the system generates the user profile. Fig. 1 presents a general scheme of Pix2Trips. Pix2Trips is a web application working on recent versions of browsers on a PC or laptop. Users do not need credentials, their identity is generated on the first visit and is kept locally (Authentication). A user is 


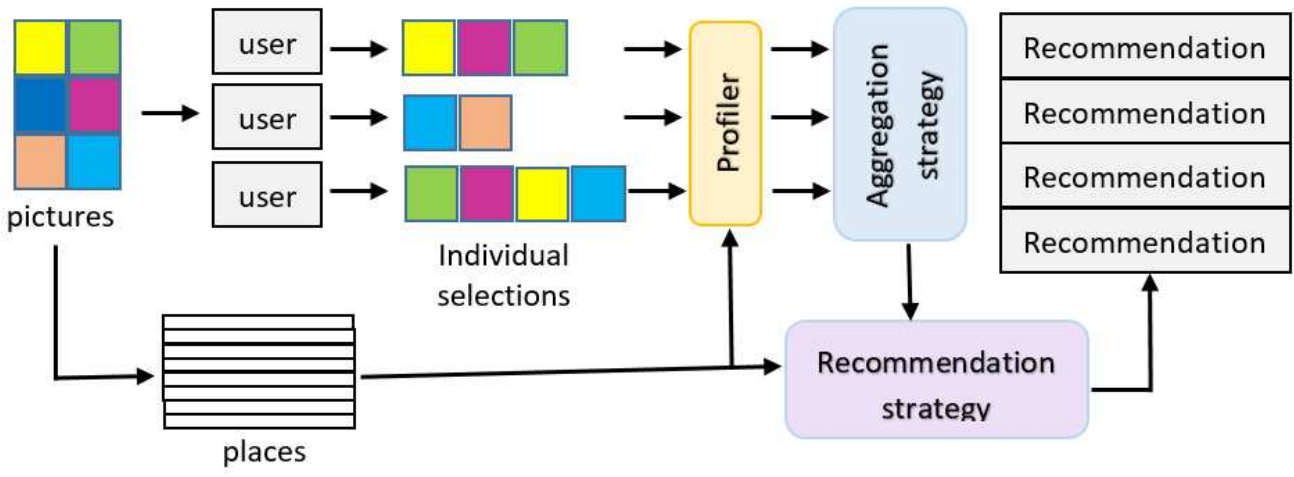

Fig. 1. A general scheme of the Pix2Trips system

represented by a name. Sessions: a session is a combination of users, individual picture selections and selected places. The group initiator creates a session, the group members join the session by a hyperlink or a unique 6-digit auto-generated code. Voting for suggestions: suggestions are derived from the users' picture selections, they are saved for later reference. Real-time interaction: users of one group belonging to one session should see each others interaction in real-time.

Specifications. To give a group of urban tourists recommendations, we need a consistent model for group preferences and places. Both have to be represented by the same set of attributes. The set of attributes depends on the tourism specificity of a given city or region (e.g., a beach). Our example is Wroclaw city. We have defined a list of 44 features used for modeling places for the recommendation. Each feature is assigned to each place with an appropriate intensity value, in scope $[0,100]$, where 0 means irrelevant feature, and 100 - the highest value. We used 39 pictures taken from a freely available popular Internet resource Unsplash at https://unsplash.com, none of them was taken in Wroclaw. The base of places for recommendation consists of 60 entries, all of them are located in Wroclaw. All places can be grouped into 11 subjects providing 44 features. The selected pictures present different objects: Museum, Sight, Church, Restaurant, etc. The list of 11 types of places and 44 features is following.

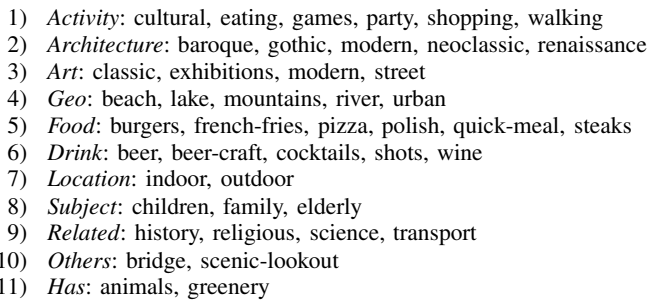

Aggregation strategies. In Pix2Trips, we implemented nine aggregation strategies, seven from literature [12], and two ours: Average + Normalize, and Composite. Below we present all aggregation methods. In all formulas, $f_{i}$ is an aggregated value of the $i$ th feature in the group; $N$ is the size of the group; $M$ is the number of features; $i$ is the feature number; $x_{k i}$ is a value of $i$ th feature of the $k$ th member of the group.

Average: it is the most straightforward and most natural strategy. Each factor is averaged between all members.

Average + Normalize : each value is normalized to 100 so the factors with highest values become the most important.

$$
f_{i}=100 \times \frac{\sum_{k=1}^{N} x_{k i} / N}{\max _{j \in[1 ; M]} \sum_{k=1}^{N} x_{k j} / N}
$$

Average without Misery: it is a standard average strategy in which values below a certain threshold are removed (set to zero). We set the threshold at $33 \%$.

Multiplicative: the values of each feature are multiplied. The strategy eliminates features unwanted by even one person.

$$
f_{i}=100 \times \frac{\prod_{k=1}^{N} x_{k i}}{\max _{j \in[1 ; M]} \prod_{k=1}^{N} x_{k j}}
$$

Least Misery: it focuses on minimizing the overall preferences, i.e. assumes that the group is as satisfied as to the least happy member.

$$
f_{i}=\min _{k \in[1 ; N]} x_{k i} \mid x_{k i}>0
$$

Most Pleasure: it acts as the opposite of Least Misery strategy, i.e., it prefers the highest values of the features.

$$
f_{i}=\max _{k \in[1 ; N]} x_{k i}
$$

Approval Voting: here, values greater than or equal to the assumed threshold are replaced by value 100, the rest is changed to zero. Next, the Average method is applied to modified vectors. We used thresholds equal to $33 \%$ and $50 \%$. Borda Count: it uses the ranks of values ordered in ascending order. If multiple features have the same value, they are assigned the same rank, but the next factor skips as many ranks as duplicates. Next, values are summed, and the resulting vector is scaled according to the maximal possible value.

Composite: it represents the ability to combine other strategies. Multiple group models are created using different strategies, next they are combined again. In Pix2Trips we implemented Multil strategy - a combination of Least Misery and Most Pleasure by Average.

Image-based preferences and recommendation strategies. The idea is based on [6], in which the preferences represent relevance to each of the seven touristic factors, but we use a different set of features. To compute the relationship between 
the pictures and the features, we apply the multivariate linear regression for each feature $f_{i}$ :

$$
f_{i}=\sum_{j=1}^{P} b_{i j} x_{j}, \quad \text { for } i \in[1, M]
$$

where $P$ is a number of pictures, and $M$ is a number of features (in Pix2Trip $P=39, M=44$ ).

We have to calculate $b_{i j}$ to determine the user's profile out of pictures. $f_{i}$ is the known numerical value representing the relevance of the feature $f_{i}$ to the place under consideration. Value of $x_{j}$ is calculated in three ways (types) [6]. Type 1: $x_{j}$ is assigned 1 if the picture has been selected and 0 otherwise. It does not use the positional information of the assigned images. Type 2: the value of $x_{j}$ for the first picture is 1 , for the 2 nd is $(M-1) / M$, according to the formula: $x_{j}=(-k+M+1) / M$ if picture $j$ is selected on $k$ th position, and 0 otherwise. Type 3: the positional information $k$ and the number of assigned pictures $n$ are considered. $x_{j}=M \frac{(-k+n+1)}{\sum_{m=1}^{n} m}$ if picture $j$ is selected on $k$ th position out of $n$, and ${ }^{m}=1$ otherwise. Once calculation the model of each place, we can determine the user profile $x_{j}^{u}$ based on the images he chose and one of the above methods (type 1, type 2 or type 3 )

Recommendation strategies. We can calculate the distance between the group's profile and all available places because both places and aggregated users' profiles are the $M$ dimensional space points: $d: \Re^{M} \times \Re^{M} \rightarrow \Re$. Vectors describing places are relatively sparse. Different distance measures can be used, in Pix2Trips, we implemented Euclidean, Manhattan and Chebyshev. In Pix2Trips, three recommendation strategies were implemented: (1)All: full-length vectors are used, (2)Non-zero: only features with strictly positive values are considered, (3)Top $N$ : profile's $\mathrm{N}$ most desired features are taken, regardless of venue

When the group model and the place description are incompatible, the distance is measured between empty vectors. In such situations, the distance is set as a constant, sufficiently large value.

\section{EXPERIMENTAL STUDY}

Pix2Trips is accessible at https://pix2trips.xyz/. Experiments were conducted on a laptop with: CPU: Intel i7-3630QM; RAM: 32GB; OS: Arch Linux5 (5.4.25-2-lts Linux kernel). Implementation of algorithms is done in Kotlin programming language (version 1.3.71), and the code is run on Java Virtual Machine 11.0.6 (OpenJDK7). The used parameters influence a set of recommended items. Each item is characterized by distance, i.e., how relevant a place is to the group profile. We performed a series of experiments to determine the best combination of system parameters. The output list of distances is aggregated into min, max, average and standard deviation to compare different parameters. In all experiments we have used a dataset consisting of 60 places in Wroclaw city, represented by ID number. Due to the limited space, we do not provide details of these experiments, only a brief overview of them and their results are mentioned. In more detail, we discuss the experiments regarding the quality of the system's recommendations, made with real participants of the study.

\section{A. Experiments with artificial groups with different character- istics}

We used three simulated groups with different degrees of shared interests (Table I). Members of group 1 have mostly common interests, group 2 has only some common interests, and group 3 have no common interests. Surprisingly, aggregation strategy Average + Normalize has the lowest distance regardless of the group. The difference in groups is visible in the Multiplicative strategy, where no commonality resulted in an empty recommendation list. Approval Voting $50 \%$ performed poorly on groups with common interests, only one recommendation was produced with this strategy for group 1 and zero for group 2. Strategy Multil, which is a composite strategy, has shown little variance between the groups. Almost all strategies took comparable time to calculate the group model ( $\approx 27 \mathrm{~ms}$.). The only outlier is Borda Count, as the algorithm for this strategy is more sophisticated $(\approx 30$ $\mathrm{ms}$.). Average + Normalize performs almost the same regardless of the recommendation strategy. The Multiplicative strategy explicitly shows what effect each strategy has: the higher distance is with strategies All, a bit smaller with Top 40. Also, Non-Zero produces a relatively high distance. Strategy Average + Normalize does not show the effect of different profile strategies. Average aggregation strategy shows that Typel and Type 2 profiles result in recommendations that are closer to the group model. Type 3 considers the assigned images, their order and the total count, resulted in the considerably higher distance between recommended places and the group profile. Generally, Average+Normalize aggregation strategy had the lowest distance, which corresponds to recommendations closest to the group profile. Changing other parameters and using the different characteristics of the artificial groups did not significantly influence the results. Based on the experiments, we can predict that configurations Average+Normalize aggregation strategy, Typel profile strategy and either Non-zero or Top 1 recommendation strategy would perform better than the others in terms of accuracy.

\section{B. Pix2Trips evaluation by users}

We asked members of seven groups of 3 or 4 members each (8 females, 14 males) - computer science students and people between 35 and 55 years old with moderate technical knowledge, to evaluate Pix2Trips. They had no prior knowledge about Pix2Trips and were familiar with the touristic places in Wroclaw to evaluate each recommendations list properly. We have used the System Usability Scale (SUS), which is a set of ten questions aiming to estimate the overall usability of a system [17]. Users assign a value between 1 (strongly disagree) and 5 (strongly agree) - known as the Likert scale. The questions are formulated alternately: positive and negative. Assigned values are adjusted that low value always corresponds to negative answer and high value - to positive. The 10 SUS questions are the following:

- SUS1. I think that I would like to use this system frequently.

- SUS2. I found the system unnecessarily complex.

- SUS3. I thought the system was easy to use.

- SUS4. I think that I would need the support of a technical person to be able to use this system. 
TABLE I

PICTURE SELECTION BY THE MEMBERS OF THREE ARTIFICIAL GROUPS

\begin{tabular}{|l|l|l|l|}
\hline$\#$ & Picture selection: Group 1 & Picture selection: Group 2 & Picture selection: Group 3 \\
\hline 1 & $4,34,22,35,11,6,13,25,31$ & $4,34,22,11,6,13,25,31,35$ & $7,36,2,22,6,21,3,9,34$ \\
2 & $6,2,33,30,17,8,23,39,28$ & $2,33,23,28,27,1,26,7,21$ & $30,4,35,28,29,19,20$ \\
3 & $35,37,39,8,40,17,3,24,13,4$ & $37,8,40,17,3,5,38,2,22$ & $11,18,6,12,17,32,13,8$ \\
4 & $32,26,19,40,34,30,23,3,4,12$ & $32,40,34,30,23,3,2,9,36$ & $24,31,14,16,37,26,33$ \\
\hline
\end{tabular}

- SUS5. I found the various functions in this system were well integrated.

- SUS6. I thought there was too much inconsistency in this system.

- SUS7. I would imagine that most people would learn to use this system very quickly.

- SUS8. I found the system very awkward to use.

- SUS9. I felt very confident using the system.

- SUS10. I needed to learn a lot of things before I could get going with this system.

According to [18], a mean score for web applications is 68 (on a scale of 1 to 100 ) - this value is our baseline for comparing our application to others with the same user interface type. Authors of [19] propose ResQue - an evaluation framework containing various questions/statements aiming to quantify different aspects of a recommender system from the endusers point of view. We focus on two aspects: the quality of recommendations and the enjoyability of the preference elicitation process. We divided the Pix2trips evaluation into two stages: in-app, and post-experiment. In the first stage, session members evaluated the system usability, i.e., the resulting list of items showing perceived accuracy on a seven point scale Worst, Awful, Poor, Fair, Good, Excellent, Best. The second part was after the group experiment. Evaluators filled out a questionnaire consisting of 10 SUS statements, six differen categories questions adopted from ResQue framework, and three questions concerning group identity [20]. A list of questions contained in the questionnaire is following:

- The 10 SUS questions

- Qual1. The recommender gave good suggestions.

- Qual2. The recommended items are diverse.

- Qual3. I found it easy to tell the system about my preferences.

- Qual4. The recommended items took my preferences into account.

- Qual5. Finding places to visit with the help of the recommender is easy.

- Qual6. If a recommender such as this one existed for other cities, I would use it to find places to visit.

- Identity1. I am happy to be a part of this group.

- Identity2. I consider my preferences similar to the rest of the group.

- Identity3. I feel the group has acted as a team.

The below scenario was given to the evaluators.

"Imagine that you, together with your friends, are planning a trip to Wroclaw. You have two full days in which you can visit and explore different places. First, you should individually choose up to 10 pictures that you most like and identify with them. After that, discuss by chat the recommendations with your group members and try to choose particular places to visit."

In questionnaires, we use a Likert scale. Following [21], we decided to use a 7-point scale in our statements, where, depending on the nature of the question, lowest possible (equal to 1) is labeled as worst imaginable / strongly disagree, middle point (4) is labeled as fair / neither, and the highest label (7) is best imaginable / strongly agree. The SUS scores were adjusted: from odd-numbered questions we subtracted 1 , answers of even-numbered questions were subtracted from 7 . The answers were added together and scaled to 100 .

All individuals score the system usability higher than the baseline (Fig. 2). The overall score for Pix2Trips is 85, which is in the 4th quartile. The highest average scores were obtained for SUS3 (Pix2Trips is uncomplicated), and SUS7 (is easy for new users). SUS2 and SUS10 scored the lowest, i.e., the system and its user interface are not complex to use. Recommendations quality received overall positive feedback.

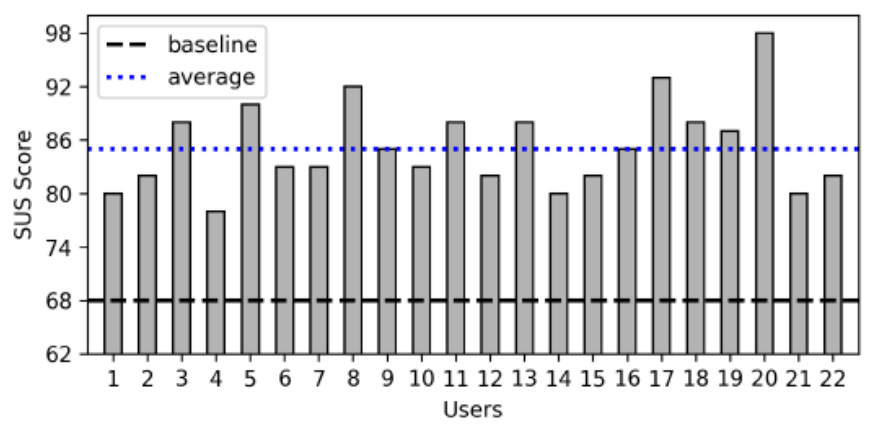

Fig. 2. SUS score per user

The users agreed that Pix2Trips generated good suggestions for their group (Qual1). Most of the evaluators assessed recommendations diversity (Qual2) as proper. 95\% of users found the specification of their preferences via pictures as easy (Qual3). According to free-form feedback, the elicitation process was often labeled as "interesting". All users found finding new places (Qual5) as easy and would use Pix2Trips for other cities (Qual6). Members of each group were happy to be together (Identity1). There was a large variance of the perceived similarity in preferences among the group members (Identity2) with $10 \%$ seeing as they have different preferences than the rest of the group. This explains the indecisive answers for Qual4, on which $41 \%$ of users put the highest score. $86 \%$ of the respondents agree that the team has acted as a team (Identity3). Users reckon that the system is beneficial for new tourists, but it could be equally useful for residents.

The in-app assessment gave us more than 350 ratings that included recommendations with different preferences and parameters, making it possible to check the correlation between distance measure and user ratings. Most ratings are around the midpoint, with the most common rating of $5(36.1 \%)$. Spearman's rank correlation coefficient $\rho=-0.110$ indicates a weak negative correlation between average distance and rating. It suggests that the distance measure is not enough to be used as a metric for recommendations quality. Most of the ratings are between midpoint (i.e., 4) and 5. Aggregation strategies Most Pleasure and Average without Misery are highest rated -4.86 and 4.85 , respectively. The classic Average was rated moderately (4.63) with the lowest standard deviation of 0.83. Our Composite aggregation strategy Multi1 has the second-lowest standard deviation at 0.89 , while the average rating is 4.56 , which places it in the middle. The distance measure is not enough good to evaluate the quality of recommendations, e.g. Average + Normalize performed best in 
our experiments but has averaged at 4.44, which is in the lower half comparing to the rest. The most controversial strategies are Multiplicative, and Approval voting 50 - their averages are the lowest at 3.95 and 4.03 , and they have the largest variance. The reason is that they often recommend only a few items. The average amount of recommended places is close to eight, in some cases, these strategies resulted in just a single or no recommendations at all. Average without Misery and Approval voting 33 produced on average 13 recommendations, due to being more aggressive to less desired features or features desired by single members. The participants mentioned that they rarely see any difference between profile strategies Type 1 and Type 2. Type 3 has produced higher average distance of recommendations -0.52 , while Type 1 and Type 2 produced very similar average distances and standard deviations.

\section{Summary}

The presented web application Pix2Trips aims to support the decision-making process of small groups of tourists who want to decide which places to visit in a new city. We have adapted an image-based preferences elicitation method for individuals to work for groups using various aggregation strategies, including two proposed in this paper. Since this is a same-time, different-place type of collaboration, real-time interactions are supported, such as messages exchange and actions connected with the recommendations themselves. The results obtained from user evaluation are promising and suggest that this approach could be used in the real world. Multiple participants in the evaluation found the preferences specification process interesting and mentioned that they would use such a system for other cities. The majority shared that their preferences were taken into account in the group recommendations, which indicates that individual preferences could be elicited by pictures and then incorporated into a group model.

We used distance as the primary measure for determining recommendations quality, which shows how close a place is to the group model. With user ratings, we chose a weak correlation between distance and perceived quality. It is not enough to determine whether one set of parameters produces a better result than another one. According to the experiments with artificial groups, the best aggregation strategy was Average + Normalize, which consistently produced recommendations closest to the group model. During the user evaluation, however, it was positioned below the average rating obtained for all recommendations. Generally, different aggregation strategies exhibit different effects that could be useful in various contexts - it could be a subject of further study. We could not determine a single best strategy to use in all cases. Average and Average without Misery were often rated above the midpoint, but the second can produce fewer recommendations than expected. Multiplicative has exhibited interesting property - the resulting places consider only the common preferences between all members. The Composite strategy used in evaluation (Multil) has been rated between Least Misery and Most Pleasure, but its rating has the lowest variance. The data preparation process could be improved because emotions evoked by pictures are somewhat subjective and can differ between groups. Pictures database has to be crafted by multiple people to achieve some level of generality. Also, assigning pictures to places should be done by experts who have visited the places under consideration. Another aspect is the process of assigning features to places. Doing it manually is tedious and errorprone, especially if multiple cities are to be supported. It would be better to use text mining and extract the information from places' descriptions and user reviews.

\section{REFERENCES}

[1] Johansen, R.: Teams for Tomorrow (groupware). In: Proc. of the Twenty-Fourth Annual Hawaii International Conf. on System Sciences. 3, Decision support and knowledge-based systems and collaboration technology, 521-534, IEEE Comput. Soc.Press (1991).

[2] Werthner, H., et al.: Future Research Issues in IT and Tourism. Information Technology \& Tourism, 15(1), 1-15 (2015)

[3] Felfernig, A. et al: Algorithms for Group Recommendation. In Group Recommender Systems, Springer Intern. Publishing, (2018) 27-58.

[4] Masthoff, J.: Group Modeling: Selecting a Sequence of Television Items to Suit a Group of Viewer. In Personalized digital television. Springer, 93-141 (2004).

[5] Nguyen, T. N.: Conversational Group Recommender Systems. In Proc. of the 25th Conf. on User Modeling, Adaptation and Personalization, 331-334 (2017).

[6] Neidhardt, J. et al: A Picture-Based Approach to Recommender Systems. Information Technology \& Tourism 15(1), 49-69 (2015). DOI: 10.1007s40558-014-0017-5.

[7] Goldberg, L. R.: An Alternative "Description of Personality": The Big Five Factor Structure. Journal of Personality and Social Psychology 59(6), 1216-1229 (1990)

[8] Gibson, H., Yiannakis, A.: Tourist Roles - Needs and the Lifecourse. Annals of Tourism Research 29(2), 358-383 (2002).

[9] Glatzer, L., Neidhardt, J., Werthner, H.: Automated Assignment of Hotel Descriptions to Travel Behavioural Patterns. In Information and Communication Technologies in Tourism, Stangl, B. and Pesonen, J., Eds., Springer, 409-421 (2018).

[10] Berger, H. et al: Quo Vadis Homo Turisticus? Towards a Picture-Based Tourist Profiler. In Information and Communication Technologies in Tourism, Sigala, M. et al. Eds., Springer, Vienna 87-96 (2007).

[11] Linaza, M. T. et al: Image-Based Travel Recommender System for Small Tourist Destinations. In Information and Communication Technologies in Tourism , Law, R. et al Eds., Springer Vienna, 1-12 (2011)

[12] Masthoff, J.: Group Recommender Systems: Combining Individual Models. In Recommender Systems Handbook, Ricci, F. et al Eds. Springer, 677-702 (2011)

[13] Ardissono, L. et al: INTRIGUE: Personalized Recommendation of Tourist Attractions for Desktop and Hand Held Devices. Applied Artificial Intelligence 17(8-9), 687-714 (2003). DOI:10.1080713827254

[14] Nguyen, T. N. and Ricci, F.: A Chat-Based Group Recommender System for Tourism. Information Technology \& Tourism 18(1-4), 5-28 (2018).

[15] Álvarez Márquez, J. O., Ziegler, J.: Hootle+: A Group Recommender System Supporting Preference Negotiation. In Collaboration and Technology, Yuizono, T. et al Eds., 9848, Springer, 151-166 (2016). DOI: 10.1007978-3-319-44799-5_12.

[16] Binucci, C. et al: Designing the Content Analyzer of a Travel Recommender System. Expert Systems with Applications 87, 199-208 (2017).

[17] Brooke, J.: SUS - A Quick and Dirty Usability Scale. In Usability evaluation in industry. CRC Press, 189-194 (1996).

[18] Bangor, A. et al: An Empirical Evaluation of the System Usability Scale. Intern. Journal of Human-Computer Interaction. 24(6), 574-594 (2008)

[19] Pu, P. and Chen, L.: A User-Centric Evaluation Framework of Recommender Systems. In Proceedings of the ACM RecSys Workshop on User-Centric Evaluation of Recommender Systems and Their Interfaces 612, 14-21, (2010).

[20] Hogg, M. A. and Hains, S. C.: Friendship and Group Identification: A New Look at the Role of Cohesiveness in Groupthink. European Journal of Social Psychology 28(3), 323-341 (1998).

[21] Dawes, J.: Do Data Characteristics Change According to the Number of Scale Points Used? An Experiment Using 5-Point, 7-Point and 10-Point Scales. International Journal of Market Research 50(1), 61-104 (2008). 